\title{
Comparison of rapid diagnostic test Plasmotec Malaria-3, microscopy, and quantitative real-time PCR for diagnoses of Plasmodium falciparum and Plasmodium vivax infections in Mimika Regency, Papua, Indonesia
}

Liony Fransisca ${ }^{1 *}$, Josef Hari Kusnanto², Tri Baskoro T Satoto³ ${ }^{3}$ Boni Sebayang ${ }^{1}$, Supriyanto ${ }^{1}$, Eko Andriyan ${ }^{1}$ and Michael J Bangs ${ }^{1}$

\begin{abstract}
Background: The World Health Organization recommends malaria be diagnosed by standard microscopy or rapid diagnostic test (RDT) before treatment. RDTs have been used with greater frequency in the absence of matching blood slide confirmation in the majority of RDT reported cases in Mimika Regency, Papua Province, Indonesia. Given the importance of RDT in current health system as point-of-care tool, careful validation of RDT product performance for providing accurate malaria diagnosis is critical.

Methods: Plasmotec Malaria-3 (XW-P07) performance was evaluated by comparing it with paired blood film microscopy and quantitative real-time PCR (qPCR). Consecutive whole blood samples were derived from one clinic in Mimika as part of routine passive malaria case detection. RDT results were read by two trained technicians and interpreted by consensus. Expert microscopic examination of blood slides was cross-checked by observer-blinded second reader and a third examiner if discordant between examinations. GPCR was used as the 'gold standard', followed by microscopy for the outcome/disease variable. Comparison analysis included sensitivity (Sn), specificity (Sp), positive and negative predictive values (PPV \& NPV), and other diagnostic screening performance measures for detecting Plasmodium falciparum and Plasmodium vivax infections.
\end{abstract}

Results: Overall malaria positive samples from qPCR was 42.2\% (175/415 samples); and from matching blood slides $40.5 \%$ (168/415) of which those infections with relatively low parasite densities $\leq 100 / \mu$ l blood was $5.7 \%$ of $P$. falciparum and $16.5 \%$ of $P$. vivax samples examined. Overall RDT performance when compared with microscopy for detecting P. falciparum was Sn:92\%, Sp:96.6\%, PPV:88\%, NPV:97.8\%, Kappa:0.87; and for P. vivax Sn:72.9\%, Sp:99.1\%, PPV:95.4\%, NPV:93.4\%, Kappa:0.79. Overall RDT performance when compared with qPCR for detecting P. falciparum was Sn:92\%, Sp:96.6\%, PPV:88\%, NPV:97.8\%, Kappa:0.87; and for P. vivax Sn:66\%, Sp:99.1\%, PPV:95.4\%, NPV:90.9\%, Kappa:0.73.

Conclusions: Plasmotec Malaria-3 test showed good overall performance scores in precision for detecting P. falciparum, but lower values regarding sensitivity and negative likelihood ratio for detecting $P$. vivax, a finding partly associated with greater frequency of lower density $P$. vivax infections compared to $P$. falciparum in this study. In particular, the negative likelihood ratio (>0.1) for $P$. vivax detection indicates RDT lacked sufficient discriminating exclusion power falling below general acceptance criteria.

Keywords: Malaria, Rapid detection test, Microscopy, PCR, Sensitivity, Specificity

\footnotetext{
* Correspondence: fransisca.liony@gmail.com

${ }^{1}$ Public Health \& Malaria Control, International SOS, PT. Freeport Indonesia,

Kuala Kencana, Papua, Indonesia

Full list of author information is available at the end of the article
} 


\section{Background}

Malaria remains a serious public health problem in Indonesia. Approximately $45 \%$ of the populations across the archipelago are at risk for malaria infection [1], with 417,819 confirmed malaria cases in 2012. However, the national annual parasite incidence (API) i.e., the number of people per 1,000 populations that experienced at least one case of malaria in a 12-month period, had decreased from $4.68 \%$ o in 1990 to $1.69 \%$ in 2012 . This is an encouraging development towards an overall national target API of below $1 \%$ by year 2030 [2].

While several areas have witnessed significant reductions in malaria prevalence, other regions (e.g., eastern Indonesia) have remained problematic for performing effective control strategies because of remoteness, lack of adequate resources and sufficient budgets to combat both vectors and parasites. Papua and West Papua provinces of Indonesia, located on the western half of the island of New Guinea, have the highest malaria burdens in the country, with recent province-wide APIs of $60.6 \%$ and 52.3\%o, respectively [3]. All four species of Plasmodium parasites are present in Papua, with Plasmodium falciparum and Plasmodium vivax as the most common infections, followed with far less frequency by Plasmodium ovale and Plasmodium malariae. In high transmission areas, mixed species infections are not uncommon. Mimika Regency, covering a vast area of the southern part of Papua Province, had an API of 531.3\%o in 2012 with an overall $P$. falciparum/P. vivax case infection ratio of 1.3:1 [4]. Other report had estimated the average API closer to $876 \%$ in the immediate Timika area, the capital of Mimika, and where the vast majority of the population resides [5].

The World Health Organization (WHO) recommends all clinically suspected malaria cases have parasitological confirmed diagnosis, using either a malaria-specific rapid diagnostic test (RDT) or direct visualization of parasites using microscopy, before treatment [6]. For more than a century, use of microscopy has been considered the 'gold standard' for malaria diagnosis, species identification, and to quantify parasitaemia [7]. Various public and private health care facilities in the Timika area can perform standard microscopic diagnosis of malaria, but this is often compromised by the poor condition and maintenance of the microscope and the irregular availability of a trained laboratory technician. In many of the remote villages in the Mimika Regency (particularly those without electricity, skilled staff, or microscopist) and most public-run clinics, only RDT is used for malaria diagnosis. INDEC Diagnostics (Jakarta, Indonesia) manufactures a multi-panel malaria RDT Plasmotec Malaria-3 (hereafter referred to using the product catalog number XW-P07) that meets ISO 13485:2003 standards [8]. Including the company's internal assessment of the RDT, there is only one known published evaluation of this product [9] that occurred in southern Sumatra; therefore, a performance evaluation of this RDT was deemed prudent if this product was to be recommended for wider use in the Mimika area. RDT quality (accuracy and precision) is especially important given the infrequent use or absence of routine microscopy or matching blood film confirmation in the majority of instances in Papua.

Among other criteria, a useful and effective RDT must have sufficiently high sensitivity to be able to accurately identify as many 'true' malaria cases as possible, especially in areas where reliable microscopy is not available or used infrequently. This is particularly important so that infections can be effectively and specifically treated based on parasite species. The screening sensitivity of an RDT can be influenced by the epidemiological characteristics and infection dynamics in the target population. As parasite antigen concentrations in the blood and parasitaemia levels can vary due to multiple host and parasite factors, the performance level of an RDT can be similarly affected depending on the malaria-endemic population involved in the product assessment [10].

Establishing test performance accuracy for disease screening should be considered before investing and committing to a specific product. Although certain tests may be relatively inexpensive and easy to use, they must be valid and provide consistent reproducible results. Test accuracy describes the diagnostic strength of the association between the predictor variable (RDT result) and outcome variable (disease) as measured against a 'gold standard' test. The most common and useful complementary measures for evaluating a test are sensitivity (the proportion of true diseased persons in a population who are test positive - the true positive rate), specificity (the proportion of truly non-diseased persons who are so identified by the test - the true negative rate), and the positive and negative predictive values (the probability that the disease is present or not when the test is positive or negative, respectively). However, unlike the previous measures, another set of complementary statistics, the positive and negative likelihood ratios, are less likely to be affected by background disease prevalence [11], and are thus considered among the best measures of test accuracy [12]. Diagnostic likelihood ratio represents the odds ratio that a positive (or negative) test will be observed in an infected population compared to the odds that the same result will be observed in a noninfected population. A positive likelihood ratio $>10$ and a negative likelihood ratio $<0.1$ generally indicate a test holds sufficient merit as a useful diagnostic tool [13]. Lastly, test consistency describes diagnostic reliability (precision) and includes a measure of agreement (Kappa statistic) between one test and another (e.g., new test versus a 'standard') using dichotomous variables (malaria 
positive or negative). A Kappa score of 1 indicates perfect agreement, while 0 indicates the equivalent of chance having produced the apparent agreement $[14,15]$.

The XW-P07 has not been listed, or data presented, as formally evaluated by the World Health Organization's Malaria RDT Product Testing Program in Rounds 1 through 5 (2008-2013) [10,16]; therefore, the objective of this study was to evaluate the performance of this device as a point-of-care diagnostic test, when compared with expert microscopy and quantitative real-time PCR (qPCR).

\section{Methods}

\section{Site and procedures}

Data collection was conducted in Kuala Kencana, a township near Timika, in April and May 2014. The health facility was selected as the XW-P07 was in current use for routine malaria diagnosis with microscopy. The annual slide positive rate (SPR) at the Kuala Kencana Clinic for 2012 and 2013 was 40\% (P. falciparum $/ P$. vivax ratio $=1.3: 1$ ) and $38 \%$ ( $P$. falciparum $/ P$. vivax $=1: 1.2$ ) respectively, indicating no significant changes in SPR while having a notable shift between the two years in the proportion of parasite species in favor of $P$. vivax. The clinic is equipped with a modern laboratory and full-time expert laboratory staff and malaria microscopists with access to detailed medical records on all malaria cases reported. The inclusion criteria for study samples included one or more of the following: 1) passive patient visit to clinic through either the emergency or outpatient department; 2) patient presenting with suspected malaria infection that included one or more of these following symptoms: fever, chills, headache, nausea, vomiting, abdominal pain and/or diarrhea, myalgia; 3) patient having lived in a malaria-endemic area or visiting one in the past four weeks; and 4) the patient may have experienced malaria before [17].

Following informed consent by patient adults or minors (with parental/legal guardian's consent), using sterile procedures, a single $3 \mathrm{ml}$ venous blood sample was collected in a glass Vacutainer tube containing $5.4 \mathrm{mg}$ dipotassium $\left(\mathrm{K}_{2}\right)$ EDTA (Becton, Dickinson and Co., New Jersey, USA) from each suspected malaria case. Blood was used to prepare an RDT with a matched thick and thin blood slide for microscopy, and qPCR testing. Approximately $1 \mathrm{ml}$ of blood was transferred into a PCR sample tube and immediately stored at $-20^{\circ} \mathrm{C}$. The RDT and first blood slide reading were recorded immediately. A second slide reading and PCR test were conducted within a week of the initial blood draw. Results based on the RDT and first blood slide reading were provided to the patient within an hour of sampling and if found infected, malaria treatment provided (artemisinin-based combination drugs and primaquine as appropriate to specific infection).
The study sampling continued in a consecutive manner until the target sample size was obtained. The minimum sample size $(n=400)$ for accurately estimating sensitivity and specificity [18] was based on the reported lowest RDT sensitivity value $(84.4 \%$ for $P$. vivax), the Mimika 2012 estimated malaria incidence of 0.53 infections per person-year in the resident population, and an absolute precision value of 0.05 . Those cases with incomplete information regarding symptom presentations or laboratory findings, and infections with only $P$. ovale or $P$. malariae parasites based on qPCR were excluded from the final analysis.

\section{Rapid diagnostic test}

The XW-P07 is a rapid, qualitative immunoassay, lateral flow cassette device that uses $5 \mu \mathrm{l}$ whole blood for the detection of $P$. falciparum-specific histidine rich protein2 (P. falciparum-HRP2), P. vivax-specific Plasmodium lactate dehydrogenase ( $P$. vivax $-\mathrm{pLDH})$, and pan-specific pLDH for all Plasmodium species (i.e., P. malariae and P. ovale).

The RDT (Batch 91155A, expiration 31 July 2015) was used according to the manufacturer's instructions provided in the product insert. Reading and interpretation of test results were done by two trained technicians and interpreted by consensus within the specified 15-30 min test window. No test was scored beyond the $30 \mathrm{~min}$ limit. Any test that failed to produce a control band was considered invalid and the test repeated. Test interpretation criteria are provided in Figure 1. As example, if matched microscopy or $\mathrm{qPCR}$ detected only $P$. vivax, while the RDT showed all four bands reactive, the interpretive result of the RDT would be P. falciparum false positive, $P$. vivax true positive, $P$. ovale and/or $P$. malariae as equivocal and undetermined. Given the low prevalence of the latter two species, this study restricted performance analysis on $P$. falciparum and $P$. vivax infections only.

\section{Microscopy}

Microscopy and qPCR were used as the reference standards in this study. With each RDT, a matching thick and thin blood film (one slide per sample) was prepared and stained with Giemsa solution (1:10 dilution for approximately $20 \mathrm{~min}$ ). Slides were examined using a compound light microscope under x1,000 oil-immersion magnification by a qualified laboratory technician in the clinic. All blood films were examined for a minimum of 100 high-magnification fields before being recorded as either negative for malaria parasites and for the detection of low density mixed species infections. The parasite densities were estimated for each sample counted separately by parasite species. Parasite numbers were reported per 200 white blood cells (WBC) to estimate 


\section{Possible result \\ Interpretation}
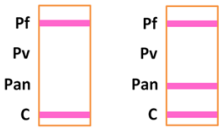

Plasmodium falciparum infection; or mixed infection with other malaria species (Plasmodium ovale or Plasmodium malariae)

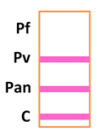

Plasmodium vivax infection; or mixed infection with other

malaria species (Plasmodium ovale or Plasmodium malariae)

Infection of other malaria species

(Plasmodium ovale or Plasmodium malariae)

Mixed infection, both Plasmodium falciparum and Plasmodium

vivax, possibly other species (undetermined).

Detection band(s) with grey color, control band with pink-purple colour. Rerun test with new kit.

Control band only.

Negative for malaria

Invalid test: No visible control line appears within $\mathbf{3 0}$ minutes.

Test again with new kit.

Figure $1 \mathrm{XW}-\mathrm{P} 07$ test criteria for determination of malaria infection.

parasite density per $\mu$ l of blood, assuming a standard mean WBC count of $8,000 / \mu$ l blood. Samples were also categorized into one of four groups based on overall parasite density $(1-100 ; 101-1,000 ; 1,001-10,000$; and 10,001-100,000 parasites/ $\mu$ l blood).

All slides were subsequently examined by an independent expert malaria microscopist as an observer-blinded cross-check and confirmation of first examination. A third, observer-blinded expert microscopist was used for slides where there was either discordant or significant discrepancies in findings (infection density or parasite species discrepancies) between the first and second examiners. In such cases, the results for the third examination were regarded as final. All microscopists in this study were experienced in malaria slide preparation and diagnosis, and certified through quality assurance procedures.

\section{Quantitative real-time PCR}

Quantitative real-time PCR (qPCR) amplification was performed using a Rotor-Gene Q (Qiagen, Hilden, Germany).
Parasite DNA (if present) was extracted from whole blood using Qiagen DNeasy Blood and Tissue Kit (Qiagen, Hilden, Germany), according to the manufacturer's instructions. Extracted DNA was eluted in a final volume of $100 \mu \mathrm{l}$ for the first elution and $50 \mu \mathrm{l}$ for the second. DNA concentration and purity were measured using a nanophotometer (Implen $\mathrm{GmBH}$, Munich, Germany) and then immediately stored at $-20^{\circ} \mathrm{C}$. Amplification was carried out in a $20 \mu \mathrm{l}$ reaction volume, containing $10 \mu \mathrm{l}$ SYBR Green (BioRad, California, USA), $0.3 \mathrm{mM}$ of each primer, 30 ng DNA template, and nuclease-free water (Promega, Wisconsin, USA). A pair of primers was used to amplify the 18S rRNA gene sequences: PL1473F18 (5'TA CGA ACG AGA TCT TAA-3') and PL1679R18 (5'GTT CCT CTA AGA AGC TTT-3') for the four Plasmodium species.

The conditions for the qPCR consisted of initial denaturation at $95^{\circ} \mathrm{C}$ for 10 minutes, 40 cycles amplification at $95^{\circ} \mathrm{C}$ for 10 seconds, $50^{\circ} \mathrm{C}$ for 5 seconds, and $72^{\circ} \mathrm{C}$ for 20 seconds each, with fluorescence acquisition at the end 
of each extension step. The melt program consisted of 2 minutes at $95^{\circ} \mathrm{C}$ and $68^{\circ} \mathrm{C}$ each, followed by a stepwise temperature increase of $0.2^{\circ} \mathrm{C} / \mathrm{s}$ until $90^{\circ} \mathrm{C}$, with fluorescence acquisition preformed at each temperature transition. Plasmodium species differentiation was achieved using melting curve analysis. Above steps and subsequent data interpretation for qPCR followed Mangold et al. [19] for detection and identification of each parasite species. A melting curve analysis with the four species controls with no template control was used in the RDT performance evaluation. Graphics were generated using default software program of Rotor-Gene Q Series Software to compare against standard melting temperatures for $P$. falciparum $75.5-77.5^{\circ} \mathrm{C}, P$. vivax $79.0-81.0^{\circ} \mathrm{C}, P$. ovale $77.5-79.0^{\circ} \mathrm{C}$, and P. malariae $73.5-75.5^{\circ} \mathrm{C}$ [19].

\section{Statistical analysis}

Data was analyzed using Stata 12.0 software (Stata Corporation, College Station, Texas, USA, license no. 08762859510). RDT performance was calculated compared with matched microscopy and qPCR results with 95\% confidence intervals (CI) for the following values: sensitivity (Sn), specificity $(\mathrm{Sp})$, positive predictive value (PPV), negative predictive value (NPV), positive likelihood ratio (PLR), negative likelihood ratio (NLR), and Kappa score. RDT sensitivity was also calculated based on parasite density. Odds ratios and confidence intervals were calculated for correlation between low parasite counts $(\leq 100 / \mu \mathrm{l}$ blood) and RDT false negative results based on matching microscopy and qPCR results; as well as between low parasite counts and body temperature at the time of blood sampling. For statistical inference, the Fisher's exact test was used for interpretation of false negative RDT results between malaria infections with densities above and below 100 parasites/ $\mu$ l blood with significance set at $p$-value of $<0.05$.

\section{Ethical review}

All observations and reporting contained herein are based on informed consent by volunteers (adults or minors with their legal guardian's consent) prior to physical and laboratory examinations. A unique medical record number was used as an identifier of each sample and all information was kept confidential throughout the study. Ethical review and clearance was obtained from the Medical and Health Research Ethics Committee (Reference no. KE/FK/320/EC), Faculty of Medicine, Gadjah Mada University, Yogyakarta, Indonesia.

\section{Results}

Between 10 April and 14 May 2014, 428 suspected malaria patients attending a local clinic in Kuala Kencana, Mimika, voluntarily provided blood samples (Figure 2). Of these initial samples, eight had incomplete data, one

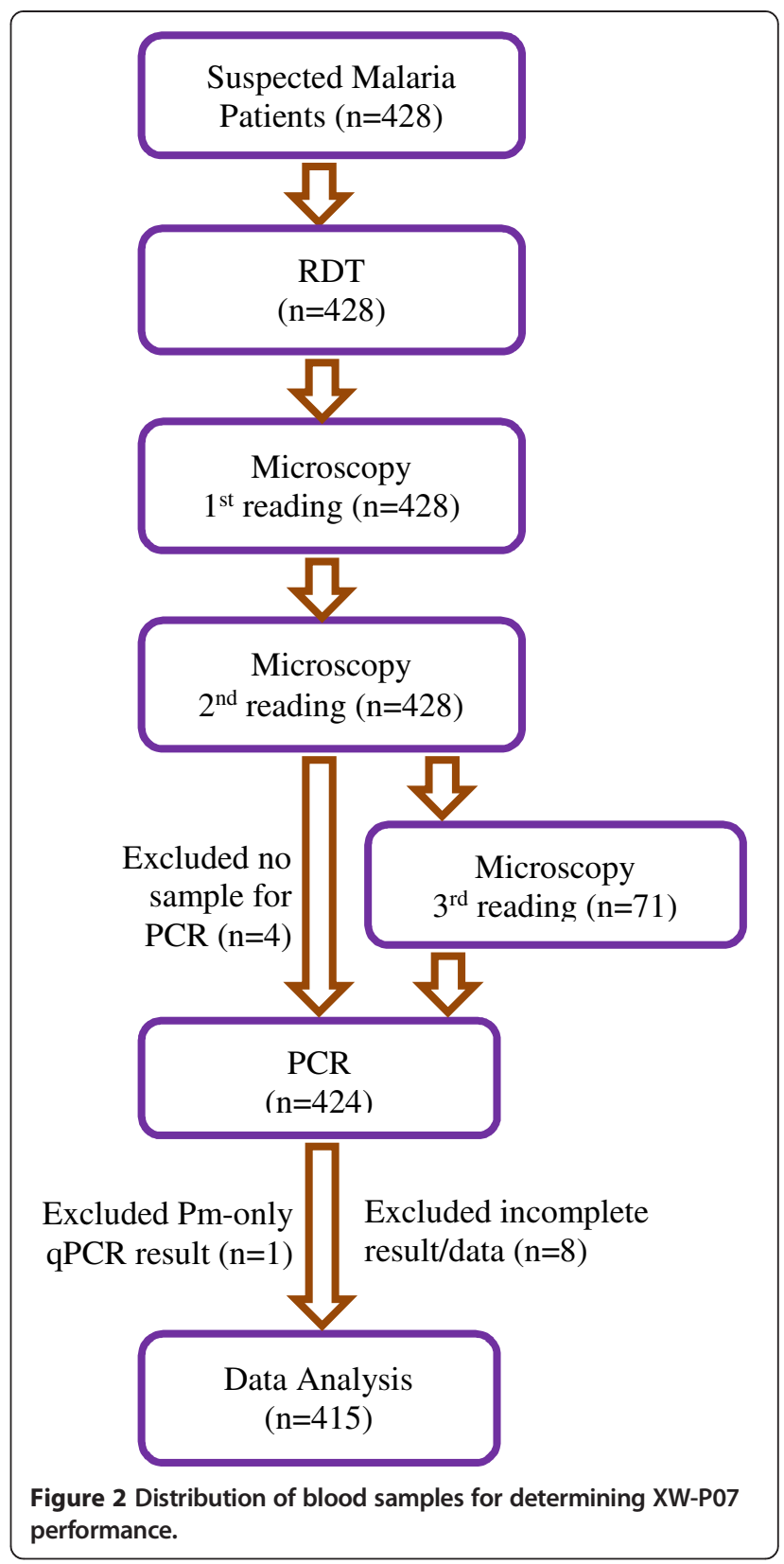

case had only $P$. malariae infection, and four lacked matching qPCR testing; therefore, only 415 samples were included in the study and analysis. No adverse events were recorded with any patient during the blood draw process or thereafter. In this study, $80 \%(\mathrm{n}=331)$ of patients reported 'fever', while only $47.5 \%(\mathrm{n}=197)$ actually had a body temperature $\geq 37.5^{\circ} \mathrm{C}$ at the time of blood sampling. Baseline characteristics of study population are shown in Tables 1 and 2.

The RDT cassettes used in this study showed clear line intensities and had good background clearing after addition of the buffer solution; however, 3 out 415 tests failed to produce a 'control' line and thus had to be 
Table 1 Baseline characteristics of analyzed samples based on microscopy diagnosis

\begin{tabular}{|c|c|}
\hline Male & $302 / 415$ (72.8\%) \\
\hline Mean age in years (range) & 28.9 years ( 6 months -76 years) \\
\hline Temperature $\geq 37.5^{\circ} \mathrm{C}$ or 'fever' & 197/398 (49.5\%) \\
\hline $\begin{array}{l}\text { Mean Plasmodium falciparum } \\
\text { parasite/ } \mu \text { l blood (range) }\end{array}$ & $11,388(40-68,040)$ \\
\hline $1-100 / \mu l$ & $5 / 88(5.7 \%)$ \\
\hline $101-1,000 / \mu l$ & 18/88 (20.5\%) \\
\hline $1,001-10,000 / \mu \mathrm{l}$ & 34/88 (38.6\%) \\
\hline $10,001-100,000 / \mu l$ & $31 / 88(35.2 \%)$ \\
\hline $\begin{array}{l}\text { Mean Plasmodium vivax } \\
\text { parasite/ } \mu \text { l blood (range) }\end{array}$ & $3,143(40-27,000)$ \\
\hline $1-100 / \mu l$ & 14/85 (16.5\%) \\
\hline $101-1,000 / \mu l$ & 25/85 (29.4\%) \\
\hline $1,001-10,000 / \mu \mathrm{l}$ & 42/85 (49.4\%) \\
\hline $10,001-100,000 / \mu l$ & $4 / 85(4.7 \%)$ \\
\hline
\end{tabular}

Positive samples were divided between $P$. falciparum and $P$. vivax infections, and those with mixed species infections were counted separately as $P$. falciparum and P. vivax.

repeated with a new cassette using the same blood sample. Laboratory test findings are shown in Table 3. RDT results found $21.0 \%(87 / 415)$ of samples reactive for $P$. falciparum, $14.5 \%(60 / 415)$ for $P$. vivax, and $1.2 \%$ (5/ 415) with mixed $P$. falciparum/P. vivax infections. Microscopy produced a total SPR of 40.5\% (168/415); with $P$. falciparum slightly more common than $P$. vivax. To determine RDT performance using matching microscopy, positive slides were divided between $P$. falciparum and $P$. vivax; and those with mixed species infections

Table 2 Signs and symptoms of suspected malaria cases with and without parasitaemia based on microscopic diagnosis

\begin{tabular}{lll}
\hline Signs and symptoms & $\begin{array}{l}\text { Parasitaemia } \\
\text { Present } \\
(\mathbf{n}=\mathbf{1 6 8})\end{array}$ & $\begin{array}{l}\text { Parasitaemia } \\
\text { Absent } \\
\mathbf{( n = 2 4 7 )}\end{array}$ \\
\hline Fever & $154(91.7 \%)$ & $177(71.7 \%)$ \\
Chills & $68(40.5 \%)$ & $52(21.0 \%)$ \\
Sweating & $13(7.7 \%)$ & $12(4.9 \%)$ \\
Nausea & $71(42.3 \%)$ & $60(24.3 \%)$ \\
Vomiting & $35(20.8 \%)$ & $32(13.0 \%)$ \\
Diarrhea & $24(14.3 \%)$ & $41(16.6 \%)$ \\
Headache & $71(42.3 \%)$ & $57(23.1 \%)$ \\
Myalgia & $52(31.0 \%)$ & $55(22.3 \%)$ \\
Paroxysm: combination chills, & $12(7.1 \%)$ & $5(2.0 \%)$ \\
fever, \& sweating & & $77(31.2 \%)$ \\
$\geq 3$ outcomes & $97(57.7 \%)$ & $26(10.5 \%)$ \\
$\geq 4$ outcomes & $60(35.7 \%)$ & $85(34.4 \%)$ \\
\hline Temp $\geq 37.5^{\circ} \mathrm{C}$ & $112(66.7 \%)$ & \\
\hline
\end{tabular}

were counted twice, separately as $P$. falciparum and $P$. vivax. Composite determinations from first, second, and third slide (if required) examinations were used to calculate final RDT performance. A total of 64 blood slides (15.4\%) out of 415 observations required a third reading for arbitration over any discrepant results from the initial first and second examinations. When compared with matched qPCR, the microscopy error rates for the first and second examinations were $12 \%$ and $8 \%$, respectively; therefore, the parasite density determinations from the second reading were used for the final analysis. Overall, compared to the RDT, microscopy resulted in an increased detection of infection, with Kappa scores (interprocedure agreement) between composite microscopy and qPCR of 0.97 for $P$. falciparum and 0.92 for $P$. vivax.

Table 4 provides final RDT performance measures and 95\% CI compared with microscopy for detecting P. falciparum was Sn 92\%, Sp 96.6\%, PPV 88\%, NPV 97.8\%, with Kappa score 0.87; and for P. vivax: Sn $72.9 \%$, Sp 99.1\%, PPV 95.4\%, NPV 93.4\%, with Kappa score 0.79 . Final RDT performance compared with qPCR for detecting P. falciparum was Sn 92\%, Sp 96.6\%, PPV 88\%, NPV 97.8\%, with Kappa score 0.87; and for P. vivax: Sn 66\%, Sp 99.1\%, PPV 95.4\%, NPV 90.9\%, with Kappa score 0.73.

RDT sensitivity for detecting $P$. falciparum or $P$. vivax was different depending on parasite density as measured from peripheral blood (Table 5). This difference was statistically significant for $P$. falciparum $(p=0.02)$ and $P$. vivax $(p<0.001)$. The RDT achieved $100 \%$ sensitivity at high parasite densities $\geq 4,800$ parasites/ $\mu$ l blood for $P$. falciparum and at a lower threshold of $\geq 640$ parasites $/ \mu \mathrm{l}$ for $P$. vivax. Parasite densities at or below $100 / \mu \mathrm{l}$ increased the probability of the RDT producing a false negative finding; thus, impacting the overall malaria detection performance of the test. When compared with microscopy, the odds ratio with low parasite densities resulting in RDT false negative findings compared to densities $>100 / \mu \mathrm{l}$ was $10.4(p=0.05)$ for $P$. falciparum, and $18(p<0.001)$ for $P$. vivax. Similarly, when compared with qPCR, the odds ratio for false negative results at low parasite densities was $6.5(p=0.2)$ for $P$. falciparum and 16.4 ( $p<0.001)$ for $P$. vivax (Table 6). In RDT comparisons, significant differences were seen with $P$. vivax compared with microscopy and qPCR, but only borderline significance with $P$. falciparum and microscopy. However, after excluding $P$. vivax densities of $\leq 100 / \mu$, the RDT performance sensitivity compared with qPCR increased from $66 \%$ (95\% CI $55.5-75.4 \%)$ to $72.8 \%$ (61.8-82.1\%), along with adjusted Sp 99.1\% (97.3-99.8\%), PPV 95.2\% (86.599\%), NPV 93.5\% (90.3-95.9\%), PLR 77.7 (25-242), NLR 0.3 (0.2-0.4), and Kappa score 0.79. Similarly, when removing the lower density $P$. falciparum infections, the RDT performance compared with qPCR remained nearly the same, increasing sensitivity only slightly from $92 \%$ 
Table 3 Summary RDT results compared with matching microscopy and qPCR in $3 \times 3$ table

\begin{tabular}{|c|c|c|c|c|c|c|c|c|c|c|c|}
\hline \multirow{3}{*}{$\begin{array}{l}\text { RDT } \\
\text { Result }\end{array}$} & \multicolumn{3}{|c|}{ Microscopy Pf } & \multirow{3}{*}{$\begin{array}{l}\text { Microscopy Pf, Pv } \\
\text { PCR } \\
\text { Pf, Pv }\end{array}$} & \multirow{3}{*}{$\begin{array}{l}\text { Microscopy Pf, Pm } \\
\text { PCR } \\
\text { Pf, Pm }\end{array}$} & \multicolumn{3}{|c|}{ Microscopy Pv } & \multicolumn{3}{|c|}{ Microscopy NP } \\
\hline & PCR & PCR & PCR & & & PCR & PCR & PCR & PCR & PCR & PCR \\
\hline & $\mathrm{Pf}$ & $\mathrm{Pf}, \mathrm{Pv}$ & NP & & & $\mathrm{Pv}$ & $\mathrm{Pv}, \mathrm{Pm}$ & NP & $\mathrm{Pf}$ & $\mathrm{Pv}$ & NP \\
\hline Pf & 32 & 0 & 0 & 1 & 0 & 0 & 0 & 0 & 0 & 0 & 8 \\
\hline Pf, Pan & 40 & 2 & 0 & 2 & 2 & 0 & 0 & 0 & 0 & 0 & 0 \\
\hline Pv & 1 & 0 & 0 & 0 & 0 & 14 & 0 & 0 & 0 & 0 & 2 \\
\hline Pv, Pan & 0 & 0 & 0 & 0 & 0 & 43 & 0 & 0 & 0 & 0 & 0 \\
\hline Pan & 1 & 0 & 0 & 0 & 0 & 0 & 0 & 0 & 0 & 0 & 0 \\
\hline Pf, Pv, Pan & 0 & 0 & 0 & 2 & 0 & 3 & 0 & 0 & 0 & 0 & 0 \\
\hline No parasites & 3 & 0 & 2 & 0 & 0 & 18 & 1 & 1 & 2 & 8 & 227 \\
\hline Eobservations & 77 & 2 & 2 & 5 & 2 & 78 & 1 & 1 & 2 & 8 & 237 \\
\hline
\end{tabular}

$\mathrm{NP}=$ No parasites. Pf: Plasmodium falciparum, Pv: Plasmodium vivax, Pm: Plasmodium malariae.

(84.3-96.7\%) to $92.9 \%$ (85.1-97.3\%), followed by Sp $96.6 \%$ (94-98.3\%), PPV 87.6\% (79-93.7\%), NPV 98.1\% (9699.3\%), PLR 27.5 (15.3-49.3), NLR 0.07 (0.03-0.16), and Kappa score 0.88 .

Normal body temperature recorded at the time of blood sampling was significantly associated with lower parasite density in both $P$. falciparum and $P$. vivax infections. The odds ratio of having a normal body temperature $\left(<37.5^{\circ} \mathrm{C}\right)$ and lower parasite densities $(\leq 100 / \mu \mathrm{l})$ was $12(95 \% \mathrm{CI}$ : 1.3-113.7; $p=0.02)$ for P. falciparum, and 5.5 (95\% CI: $1.6-18.9 ; p=0.004)$ for $P$. vivax.

\section{Discussion}

Definitive diagnosis and confirmation of disease status is the cornerstone of evidence-based medicine. Many malariaendemic areas of the world lack sufficient capacity and resources to accurately diagnose the infection and where reliance on the presentation of clinical signs and symptoms alone are inadequate and imprecise indicators of specific disease. The WHO recommendations for procurement of malaria RDTs are currently based on the attainment of a set of minimum performance criteria (e.g., detection rate/panel detection score, specificity, invalid rate, etc.) in the WHO Malaria RDT Product Testing Program [16] and recommendations established by the WHO Malaria Policy Advisory Committee in 2012 [20]. Products that fail to meet the full set of minimum performance criteria are not eligible for procurement by WHO. To some degree, many other organizations and government procurement authorities also follow the WHO guidelines for product selection. Based on published findings [10,16], the XW-P07 RDT has not been tested by the standardized, laboratory-based WHO program; therefore, an evaluation regarding its performance for detection of malaria compared to microscopy and $\mathrm{qPCR}$ in a point-of-care operational setting was deemed prudent and essential.

The RDT cassettes were easy to use and provided distinct, easy to interpret test lines. In only 3 tests did the RDT fail to show a control line, producing an invalid rate' of $0.72 \%$, well within the acceptable limit $(<5 \%)$ established by WHO [16]. The RDT showed sensitivity and specificity values of $>90 \%$, PLR $>10$, NLR $<0.1$, and

Table 4 RDT performance compared with matching microscopy and qPCR $(n=415)$ for all infections regardless of parasite density

\begin{tabular}{|c|c|c|c|c|}
\hline RDT performance & $\begin{array}{l}\text { Plasmodium falciparum } \\
(\mathrm{n}=\mathbf{8 8} \text { microscopy }+)\end{array}$ & $\begin{array}{l}\text { Plasmodium vivax } \\
(\mathrm{n}=85 \text { microscopy }+)\end{array}$ & $\begin{array}{l}\text { Plasmodium falciparum } \\
(\mathrm{n}=\mathbf{8 8} \mathrm{qPCR}+)\end{array}$ & $\begin{array}{l}\text { Plasmodium vivax } \\
(\mathrm{n}=94 \mathrm{qPCR}+)\end{array}$ \\
\hline Prevalence & $21.2 \%$ & $20.5 \%$ & $21.2 \%$ & $22.6 \%$ \\
\hline $\mathrm{Sn}^{*}$ & $92 \%(84.3-96.7 \%)$ & $72.9 \%(62.2-82 \%)$ & $92 \%(84.3-96.7 \%)$ & $66 \%(55.5-75.4 \%)$ \\
\hline$S p^{*}$ & $96.6 \%$ (94.1-98.3\%) & $99.1 \%(97.4-99.8 \%)$ & $96.6 \%(94.1-98.3 \%)$ & $99.1 \%(97.3-99.8 \%)$ \\
\hline$P P V^{*}$ & 88\% (79.6-93.9\%) & $95.4 \%(87.1-99 \%)$ & $88 \%$ (79.6-93.9\%) & $95.4 \%(87.1-99.0 \%)$ \\
\hline$N P V^{*}$ & $97.8 \%$ (95.6-99.1\%) & $93.4 \%$ (90.3-95.8\%) & $97.8 \%$ (95.6-99.1\%) & $90.9 \%(87.3-93.7 \%)$ \\
\hline$P L R^{*}$ & $27.4(15.3-49.1)$ & $80.2(25.8-249)$ & $27.4(15.3-49.1)$ & $70.6(22.7-220)$ \\
\hline$N L R^{*}$ & $0.08(0.04-0.17)$ & $0.27(0.19-0.39)$ & $0.08(0.04-0.17)$ & $0.34(0.26-0.46)$ \\
\hline Kappa & 0.87 & 0.79 & 0.87 & 0.73 \\
\hline
\end{tabular}

$\mathrm{Sn}=$ sensitivity; $\mathrm{Sp}=$ specificity; $\mathrm{PPV}=$ positive predictive value; NPV = negative predictive value; $\mathrm{PLR}=$ positive likelihood ratio; $\mathrm{NLR}=$ negative likelihood ratio. *95\% Cl. 
Table 5 RDT percent test sensitivity by parasite density based on microscopy for Plasmodium falciparum and Plasmodium vivax

\begin{tabular}{|c|c|c|c|c|}
\hline \multirow[t]{2}{*}{ Parasite density in blood } & \multicolumn{2}{|l|}{ Plasmodium falciparum } & \multicolumn{2}{|l|}{ Plasmodium vivax } \\
\hline & $\mathrm{n}=\mathrm{RDT}+$ /microscopy + & Sn (\%) & $\mathrm{n}=\mathrm{RDT}+/$ microscopy + & Sn (\%) \\
\hline $1-100 / \mu l$ & $3 / 5$ & 60 & $3 / 14$ & 21.4 \\
\hline $101-1,000 / \mu l$ & $14 / 18$ & 77.8 & $13 / 25$ & 52 \\
\hline $1,001-10,000 / \mu l$ & $33 / 34$ & 97 & $42 / 42$ & 100 \\
\hline $10,001-100,000 / \mu l$ & $31 / 31$ & 100 & $4 / 4$ & 100 \\
\hline Cut-off for $100 \%$ Sensitivity & 4,800 parasites/ $\mu \mathrm{l}$ & & 640 parasites $/ \mu l$ & \\
\hline
\end{tabular}

$\mathrm{Sn}=$ sensitivity.

Kappa $>0.8$ for detecting $P$. falciparum infections when compared with microscopy and qPCR. On the other hand, for $P$. vivax, the RDT showed the same specificity $>90 \%$ and PLR >10; while the overall sensitivity was much lower when compared with microscopy and qPCR (73\% and 66\% respectively), with NLR >0.1 and Kappa score slightly $<0.8$. The test specificity for both parasite species easily met the WHO recommended minimum performance criteria of $>90 \%$ (i.e., less than $10 \%$ false positive rate) detection at 200 parasite/ $\mu \mathrm{l}$ [20].

An unpublished product evaluation by the XW-P07 manufacturer reported findings from 251 samples compared to microscopy (SPR 16.3\%) showing Sn and Sp of $100 \%$ for both $P$. falciparum and $P$. vivax [8]. A study performed in Lampung Province, Sumatra, with 400 samples (SPR 36\%) showed Sn 91\% (85-97\%), Sp 99\% (98-100\%), PPV 98\% (95-100\%), NPV 97\% (95-99\%) for P. falciparum; and Sn 84\% (75-92\%), Sp 100\%, PPV 100\%, NPV 96\% (94-98\%) for P. vivax based on comparisons with matched microscopy [9]. The study in Mimika demonstrated different predictive values for both parasites compared to previous investigations which may have been influenced by the different disease prevalence in each

Table 6 Odds ratios of RDT false negative results

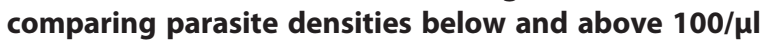
blood for Plasmodium falciparum and Plasmodium vivax

\begin{tabular}{|c|c|c|c|c|}
\hline Microscopy & $\begin{array}{l}\text { False } \\
\text { Negative }\end{array}$ & $\begin{array}{l}\text { True } \\
\text { Positive }\end{array}$ & Odds ratio $(95 \% \mathrm{Cl})$ & $p$-value \\
\hline$P f \leq 100 / \mu l$ & 2 & 3 & $10.4(1.4-77.2)$ & 0.05 \\
\hline Pf $>100 / \mu l$ & 5 & 78 & & \\
\hline$P V \leq 100 / \mu l$ & 11 & 3 & $18.0(4.4-74.5)$ & $<0.001$ \\
\hline PV $>100 / \mu l$ & 12 & 59 & & \\
\hline \multirow[t]{2}{*}{ qPCR } & False & True & Odds ratio $(95 \% \mathrm{Cl})$ & $p$-value \\
\hline & Negative & Positive & & \\
\hline$P f \leq 100 / \mu l$ & 1 & 3 & $6.5(0.5-77.3)$ & 0.2 \\
\hline$P f>100 / \mu l$ & 4 & 78 & & \\
\hline$P \vee \leq 100 / \mu l$ & 10 & 3 & $16.4(3.9-68.6)$ & $<0.001$ \\
\hline PV $>100 / \mu l$ & 12 & 59 & & \\
\hline
\end{tabular}

Pf: Plasmodium falciparum, Pv: Plasmodium vivax. study [21]. Any direct comparison between studies on RDT performance may be compromised by other factors related to location, sample population, background malaria exposure, and degree of acquired partial immunity in the target populations. This study showed that RDT sensitivity is clearly influenced by parasite density, not an unexpected finding based on testing of other products $[10,16]$. RDTs have been shown to produce lower sensitivity in areas with more frequent low parasite densities [22,23]. In this study, body temperature was significantly associated with parasite density as measured in peripheral blood normal temperatures at time of exam produce lower infection densities while corroborating other observations that a rise in body temperature is correlated with an increase in parasite density $[24,25]$.

This product evaluation was not without some underlying limitations. Firstly, this study only used RDT cassettes from a single lot number; thus, possible inter-lot variability in performance between product production periods was not assessed. This study was conducted in a reasonably controlled setting with trained laboratory technicians; therefore, extrapolation of findings to areas under more demanding environmental conditions and clinical expertise (e.g., remote primary health care clinics) should be made with caution. Albeit relatively uncommon infections, $P$. ovale and $P$. malariae were not specifically included in the panel assay (only as a pan-specific pLDH for all Plasmodium spp.); however, the majority of these infections in Papua are often coincident (mixed) with other plasmodial species. In this study, three P. malariae infections were either mixed with $P$. falciparum (two cases) or P. vivax (one case). Lastly, a set minimum of 100 high magnification fields were used for blood examination of films which possibly contributed to the relatively high (15.4\%) discordant results between the first 2 microscopists. The detection accuracy would have likely been enhanced had each reader examined a minimum of 200 fields.

Various host and parasite factors are possible reasons for varying RDT performance values between different malaria endemic populations [10,26-31]. Greater sensitivity is a desired attribute and maybe more important 
compared to test specificity to ensure malaria infections are correctly diagnosed and promptly treated to avoid development of disease complications and more severe infections when left untreated. Undetected cases due to false negative results also contribute a continuing source of gametocyte carriers (reservoirs) for sustaining malaria transmission in an area [31]. Conversely, higher test sensitivity may result in lower test specificity (higher false positive results), thereby increasing unnecessary malaria treatments [32]. Typically, P. falciparum infections have been regarded as the only human plasmodial species responsible for common causes of severe morbidity and mortality; however, that general perception has changed and been challenged by a number of recent studies showing that $P$. vivax can produce substantially greater morbidity manifesting as severe infections, causing acute and chronic anaemia, and ultimately resulting in death [33-36]. It is because of these heightened concerns regards the higher likelihood of more severe outcomes caused by $P$. vivax infections, that accurate diagnosis of this species becomes an even greater priority to ensure early and effective treatment. An RDT that lacks the necessary sensitivity for detecting $P$. vivax ( $>90 \%$ preferred) and poor exclusion power (a negative diagnostic likelihood ratio of $>0.1$ ) to adequately rule out infection presents a distinct disadvantage to both patient and health care provider in areas where the parasite is common.

This study showed that XW-P07 has a significantly lower detection rate for $P$. vivax than for P. falciparum, even when excluding low density infections below 100 parasites per $\mu \mathrm{l} /$ peripheral blood. Published WHO product evaluation on different malaria RDT products submitted for testing has shown that targeting P. falciparum HRP2 has the highest and most consistent detection rate [16]. However, this conflicts with other findings in which HRP2-based RDTs have shown a lower performance value than products using $\mathrm{pLDH}$ for detecting $P$. falciparum, as the pLDH capture system is not affected by a possible 'prozone' effect, parasite antigen polymorphisms or gene deletions [37-41]. In Myanmar, a study comparing a commonly used RDT utilizing HRP2 and pan pLDH compared with microscopy demonstrated $P$. vivax and $P$. malariae were detected to a far lesser extent (lower sensitivity, NPV, and NLR) than P. falciparum [42]. For $P$. vivax detection, separate aldolase and pLDH targeting RDTs have been shown to perform differently depending on the samples tested, increasing the risk of misdiagnosis and therefore suggesting that test sensitivity for $P$. vivax can be improved by using a combination of both aldolase and pLDH in a single RDT [43]. The RDT evaluated in this study only utilizes pLDH for detection of $P$. vivax, thus one possible explanation for the inferior sensitivity seen. The XW-P07 showed considerably lower sensitivity and a poor negative likelihood ratio, both measures falling below general acceptance criteria for detecting $P$. vivax infections. This is especially problematic for infections presenting with lower parasitaemia; thus, RDT results with such limitations must be interpreted with caution if the test is the sole method of diagnosis, particularly given the importance and high prevalence of vivax malaria in the Mimika area.

Furthermore, as a four-band RDT with one control line and three test lines (Figure 1), the XW-P07 relies on more antigen/antibody reactions using a single buffer compared to other RDTs with only three indicator bands. In areas where the prevalence of $P$. ovale and $P$. malariae is relatively low, a three-band RDT with better overall performance and able to differentiate $P$. falciparum and P.vivax, or possibly a P. falciparum/Pan-malaria test, may be a better format. Whenever possible, it would also be prudent and strongly advised to back-up all RDT diagnosis, regardless of RDT performance rating, with matched blood films and proficient microscopic examination.

A recent study in Flores, Indonesia, found that qPCR revealed almost eight times more Plasmodium infections when compared with microscopy, taking into account the high number of sub-microscopic infections in a relatively low transmission area [44]. Molecular methods are universally accepted as more sensitive than microscopy alone. However, PCR (e.g., multiplex, real-time or conventional) requires a sophisticated laboratory setting, trained technicians, entails a longer diagnosis time and higher costs to support the system; thereby precluding its routine use in most malaria endemic areas of the world - Indonesia and Mimika included. New or improved diagnostic methods are in development [45-48] that may vastly improve diagnostic capabilities and accuracy in challenging locations and basic health care settings. However, until superior, easy-to-use alternatives are available, both the RDT and microscopy, alone or in combination, will remain the mainstays for routine point-of-care malaria diagnosis.

In areas with high prevalence of $P$. vivax infection, from a cost-effectiveness point of view, standard expert microscopy should continue to be used as the reference gold standard for malaria diagnosis despite the likelihood of missing some low density parasitemia and submicroscopic infections. With skilled technicians and experienced health care providers, microscopy has more than sufficient, if not excellent, diagnostic capacity in most instances. All public-funded health facilities and private clinics in the Mimika Regency must either begin, or ensure the continuation of microscopy, as their primary means of malaria diagnosis. Microscopy should be used for routine confirmation of all RDTs performed in clinical settings whenever possible. Lastly, in many circumstances without external funding to support procurement and routine access to RDTs, the sustainability 
for maintaining these devices in all clinics is vulnerable to supply disruptions without adequate safeguards and reliable logistical support in place. The use of microscopy, even absent the aid of electricity in the most remote areas, is a sustainable approach and within the supportive framework of the Indonesian health care system. Moreover, providing basic electrical power using efficient solar capture devices and battery storage for a microscope and basic clinical equipment is well within the means of most local health budgets. The availability and routine use of microscopy also enables a facility to diagnose other important endemic diseases (e.g., tuberculosis, lymphatic filariasis, intestinal helminths and protozoa) and hematological conditions and indicators without the need of more sophisticated and costly techniques and medical instrumentation.

As malaria represents one of the leading and arguably most important health concern in the Mimika area, various health program stakeholders should continue or adopt a policy of investment in the procurement and maintenance of quality microscopes, the recruitment of additional trained laboratory technicians, and organize regular refresher training on microscopy and RDT proficiency. Nevertheless, for logistical and operational rationale, the use of RDTs will continue to play a valuable and important role in remote areas for first line diagnosis of malaria. Health care facilities in remote locations with limited laboratory capacity should continue to use high quality malaria RDTs combined with evidence from good clinical observations until microscopy can be introduced.

\section{Conclusions}

As a point-of-care device, the XW-P07 provided good test sensitivity, specificity, positive and negative predictive values and likelihood ratios, as well as inter-procedure agreement for detecting $P$. falciparum infections. For $P$. vivax infections, the test provided acceptable specificity and positive likelihood ratio, but with lower sensitivity, negative likelihood ratio (below test acceptance criteria), and inter-procedure agreement comparison. Low parasite blood densities ( $\leq 100$ parasites/ $\mu \mathrm{l})$, especially with $P$. vivax, increased the probability of false negative test results. Normal body temperature was strongly associated with the incident of lower parasitaemia, further complicating diagnosis. The RDT meets WHO minimum performance criteria for test specificity $(>90 \%)$ and invalid rate $(<5 \%)$. As vivax malaria is a very common and important parasitic infection in the population studied, all primary health centers in the Mimika Regency should begin, or continue using, expert-level and quality assured standard microscopy in a sustainable manner for greater accuracy in malaria detection.

\section{Competing interests}

The authors received no product or financial support outside of normal operating budgets. The authors declare that they have no competing interests.

\section{Authors' contribution}

The study was conceptualized and designed by LF and MJB. LF, BS, S, and EA organized sample collection and performed data entry. LF performed statistical analysis. LF, MJB, BS analyzed and interpreted the results and drafted the manuscript. JHK, TBTS, and MJB provided major inputs for the manuscript revision and finalization. All authors approved the final manuscript.

\section{Acknowledgements}

We thank Haripurnomo Kushadiwijaya, Edi Harianto, Vonny Hartana, and laboratory staff for their kind contributions to data collection and technical support. We are also grateful to PT. Freeport Indonesia, an affiliate of Freeport-McMoRan Inc., for the continued support towards improvement of the health and welfare in their workforce and local communities.

\section{Author details}

${ }^{1}$ Public Health \& Malaria Control, International SOS, PT. Freeport Indonesia, Kuala Kencana, Papua, Indonesia. ${ }^{2}$ Center for Tropical Medicine, Faculty of Medicine, Gadjah Mada University, Yogyakarta, Indonesia. ${ }^{3}$ Public Health Department, Faculty of Medicine, Gadjah Mada University, Yogyakarta, Indonesia.

Received: 1 October 2014 Accepted: 20 February 2015

Published online: 05 March 2015

\section{References}

1. Indonesia Ministry of Health. Bersama Kita Berantas Malaria. Jakarta: $\mathbf{I M O H}$; 2010 [http://www.depkes.go.id/index.php?vw=2\&id=1055]

2. Indonesia Ministry of Health. Eliminasi Malaria di Indonesia. Keputusan Menteri Kesehatan RI No. 293/MENKES/ SK/V/2009. Jakarta: IMOH; 2009 [http://pppl.depkes.go.id]

3. Indonesia Ministry of Health. Indonesia Health Data and Profile. Jakarta: Center of Data and Information; 2012 [http://www.depkes.go.id]

4. Mimika Health Office. Annual Report of Malaria. Timika: Disease Control and Environmental Unit; 2012.

5. Karyana M, Burdarm L, Yeung S, Kenangalam E, Wariker N, Maristela R, et al Epidemiology of multidrug resistant $P$. vivax and $P$. falciparum infection in southern Papua, Indonesia. Malar J. 2008;7:148.

6. Centers for Disease Control and Prevention. Diagnosis and Treatment of Malaria in the Malaria-endemic World. Atlanta: Division of Parasitic Diseases and Malaria; 2012 [http://www.cdc.gov/malaria/malaria_worldwide/reduction/ dx_tx.html]

7. Centers for Disease Control and Prevention. Malaria Diagnosis Microscopy. Atlanta: Division of Parasitic Diseases and Malaria; 2010 [http://www.cdc. gov/malaria/diagnosis_treatment/microscopy.html]

8. Diagnostics INDEC. Plasmotec Malaria-3. Jakarta: INDEC Diagnostics; 2012 [http://indec-diagnostics.co.id/?q=en/product/plasmotec-malaria-3]

9. Sutanto I, Siregar R, Astuty H, Yuwanto F, Setyaningrum E, Dodi K, et al. Penggunaan uji cepat Plasmotec Malaria-3 untuk diagnosis penderita malaria akut di Puskesmas Hanura, Lampung Selatan. Medika. 2006;32:540-5.

10. World Health Organization. Malaria Rapid Diagnostic Test Performance Result of WHO Product Testing of Malaria RDTs Round 1. Geneva: World Health Organization; 2009.

11. University of Oxford. Likelihood Ratios. Oxford: Center for Evidence-Based Medicine; 2014 [http://www.cebm.net/likelihood-ratios/]

12. McGee S. Simplifying likelihood ratios. J Gen Intern Med. 2002;17:647-50.

13. Jaeschke R, Guyatt G, Sackett D. Users' Guide to the Medical Literature. III. How to use an article about a diagnostic test. B. What are the results and will they help me in caring for my patients? The Evidence-Based Medicine Working Group. J Am Med Assoc. 1994;271:703-7.

14. Kanchanaraksa S. Evaluation of Diagnostic and Screening Tests Validity and Reliability. Baltimore: John Hopkins University; 2008 [http://ocw.jhsph.edu/ courses/fundepi/PDFs/Lecture11.pdf]

15. Viera A, Garrett J. Understanding interobserver agreement, the kappa statistic. Fam Med. 2005;37:360-3. 
16. World Health Organization. Malaria Rapid Diagnostic Test Performance: Summary Result of WHO Product Testing of Malaria RDTs Round 1-5. Geneva: World Health Organization; 2012.

17. Indonesia Ministry of Health. Gebrak Malaria - Pedoman Penatalaksanaan Kasus Malaria di Indonesia. Jakarta: Center of Disease Control; 2008.

18. Malhotra R, Indrayan A. A simple nomogram for sample size for estimating sensitivity and specificity of medical tests. Indian J Ophthalmol. 2010;58:519-22.

19. Mangold K, Manson R, Koay E, Stephens L, Regner M, Thomson R, et al. Real-Time PCR for detection and identification of Plasmodium spp. J Clin Microbiol. 2005;43:2435-40.

20. World Health Organization. WHO Global Malaria Programme Information Note on Recommended Selection Criteria for Procurement of Malaria Rapid Diagnostic Tests (RDTs). Geneva: World Health Organization; 2012.

21. Parikh R, Mathai A, Parikh S, Sekhar C, Thomas R. Understanding and using sensitivity, specificity, and predictive value. Indian J Ophthalmol. 2008;56:45-50.

22. Shakya G, Gupta R, Pant SD, Poudel P, Upadhaya B, Sapkota A, et al. Comparative study of sensitivity of rapid diagnostic (hexagon) test with calculated malarial parasitic density in peripheral blood. J Nepal Health Res Council. 2012:10:16-9.

23. Kim SH, Nam MH, Roh KH, Park HC, Nam DH, Park GH, et al. Evaluation of rapid diagnostic test specific for Plasmodium vivax. Trop Med Int Health. 2008;13:1495-500.

24. Ejezie G, Ezedinachi E. Malaria parasite density and body temperature in children under 10 years of age in Calabar, Nigeria. Trop Geogr Med. 1992:44:97-101.

25. Gravenor M, Lloyd A, Kremsner P, Missinous M, English M, Marsh K, et al. A model for estimating total parasite load in Falciparum malaria patients. J Theor Biol. 2002;217:137-48

26. Rosanas-Urgell A, Lin E, Manning L, Rarau P, Laman M, Senn N, et al. Reduced risk of Plasmodium vivax malaria in Papua New Guinean children with Southeast Asian ovalocytosis in two cohorts and a case-control study. PLoS Med. 2012;9:e1001305.

27. Cattani J, Gibson F, Alpers M, Crane G. Hereditary ovalocytosis and reduced susceptibility to malaria in Papua New Guinea. Trans R Soc Trop Med Hyg. 1987:81:705-9.

28. Allen SJ, O'Donnell A, Alexander NDE, Mgone CS, Peto TEA, Clegg JB, et al. Prevention of cerebral malaria in children in Papua New Guinea by Southeast Asian ovalocytosis band 3. Am J Trop Med Hyg. 1999;60:1056-60.

29. Fortin A, Stevenson MM, Gros P. Susceptibility to malaria as a complex trait: Big pressure from a tiny creature. Oxf J Human Mole Genet. 2002;11:2469-78.

30. Cockburn IA, Mackinnon MJ, O'Donnell A, Allen SJ, Moulds JM, Baisor M, et al. A human complement receptor-1 polymorphism that reduces Plasmodium falciparum rosetting confers protection against severe malaria. Proc Natl Acad Sci U S A. 2004;101:272-7.

31. Cheng Q, Gatton M, Barnwell J, Chiodini P, McCarthy J, Bell D, et al. Plasmodium falciparum parasites lacking histidine-rich protein 2 and 3: a review and recommendations for accurate reporting. Malar J. 2014;13:283.

32. World Health Organization. Guidelines for the Treatment of Malaria. 2nd ed. Geneva: World Health Organization; 2010 [http://whqlibdoc.who.int/ publications/2010/9789241547925_eng.pdf]

33. Sarkar D, Ray S, Saha M, Chakraborty A, Talukdar A. Clinico-laboratory profile of severe Plasmodium vivax malaria in a tertiary care center in Kolkata. Trop Parasitol. 2013;3:53-7.

34. Barcus MJ, Basri H, Picarima H, Manyakori C, Sekartuti, Elyazar l, et al. Demographic risk factors for severe and fatal vivax and falciparum malaria among hospital admissions in northeastern Indonesian Papua. Am J Trop Med Hyg. 2007;77:984-91.

35. Tjitra E, Anstey NM, Sugiarto P, Warikar N, Kenangalem E, Karyana M, et al. Multidrug-resistant Plasmodium vivax associated with severe and fatal malaria: a prospective study in Papua, Indonesia. PLoS Med. 2008;5:e128.

36. Genton B, D'Acremont V, Rare L, Baea K, Reeder JC, Alpers PA, et al. Plasmodium vivax and mixed infections are associated with severe malaria in children: a prospective cohort study from Papua New Guinea. PLoS Med. 2008:5:e127.

37. Maltha J, Gamboa D, Bendezu J, Sanchez L, Cnops L, Gillet P, et al. Rapid diagnostic tests for malaria diagnosis in the Peruvian Amazon: impact of phrp2 gene deletions and cross-reactions. PLoS One. 2012;7:e43094.

38. Gillet P, Scheirlinck A, Stokx J, De WA, Chauque HS, Canhanga OD, et al Prozone in malaria rapid diagnostics tests: how many cases are missed? Malar J. 2011:10:166.
39. Baker J, Ho MF, Pelecanos A, Gatton M, Chen N, Abdullah S, et al. Global sequence variation in the histidine-rich proteins 2 and 3 of Plasmodium falciparum: implications for the performance of malaria rapid diagnostic tests. Malar J. 2010:9:129.

40. Kumar N, Singh JP, Pande V, Mishra N, Srivastava B, Kapoor R, et al. Genetic variation in histidine rich proteins among Indian Plasmodium falciparum population: possible cause of variable sensitivity of malaria rapid diagnostic tests. Malar J. 2012;11:298.

41. Maltha J, Guiraud I, Lompo P, Kabore B, Gillet P, Geet C, et al. Accuracy of $P$. falciparum HRP2 versus $P$. falciparum $\mathrm{pLDH}$ antigen detection by malaria rapid diagnostic tests in hospitalized children in a seasonal hyperendemic malaria transmission area in Burkino Faso. Malar J. 2014;13:20.

42. Kosack C, Naing WT, Piriou E, Shanks L. Routine parallel diagnosis of malaria using microscopy and the malaria rapid diagnostic test SD 05FK60: the experience of Medecins Sans Frontieres in Myanmar. Malar J. 2013;12:167.

43. Dzakah E, Kang K, Ni C, Tang SX, Wang JH, Wang JF. Comparative performance of aldolase and lactate dehydrogenase rapid diagnostic tests in Plasmodium vivax detection. Malar J. 2014;13:272.

44. Kaisar M, Supali T, Wiria A, Hamid F, Wammes L, Sartono E, et al. Epidemiology of Plasmodium infection in Flores Island, Indonesia using realtime PCR. Malar J. 2013;12:169.

45. Mori Y, Kanda H, Notomi T. Loop-mediated isothermal amplification (LAMP): recent progress in reasarch and development. J Inf Chemother. 2013;19:404-11.

46. Cook J, Aydin-Schmidt B, González IJ, Bell D, Edlund E, Nassor MH, et al. Loop-mediated isothermal amplification (LAMP) for point-of-care detection of asymptomatic low-density malaria parasite carriers in Zanzibar. Malar J. 2015;14:43.

47. Orban A, Butykai A, Molnar A, Prohle Z, Fulop G, Zelles T, et al. Evalution of a novel magneto-optical method for the detection of malaria parasites. PLoS One. 2014;9:e96981.

48. Mello M, Luz F, Leal-Santos F, Alves Jr E, Gasquez T, Fontes C. Standardization of blood smears prepared in transparent acetate: an alternative method for the microscopic diagnosis of malaria. Malar J. 2014;13:238.

\section{Submit your next manuscript to BioMed Central and take full advantage of:}

- Convenient online submission

- Thorough peer review

- No space constraints or color figure charges

- Immediate publication on acceptance

- Inclusion in PubMed, CAS, Scopus and Google Scholar

- Research which is freely available for redistribution 